\title{
Quelques caractéristiques anatomiques des systèmes nerveux et musculaire du membre thoracique du Chameau
}

\author{
par M.-A.-A. EL HAGRI, F.R.C.V.S., B.V.SC. et M.-B. MORCOS, B.V.SC.
}

La litterature concernant l'anatomie du chameau est réduite et loin d'être complète et pleinement instructive. Ce défaut d'informations adéquates nous a incités à effectuer dans notre Service quelques recherches poussées ayant pour objet de reculer les limites de nos connaissances en ce domaine. La présente étude fait partie de ce programme de recherches.

\section{A. - LE SYSTÈME NERVEUX}

Lesbre (1903) a donné une très brève description du plexus brachial et en a nommé les ramifications. Parmi celles-ci, il a brièvement décrit le nerf cubital et le nerf médian. Il établit que ce demier, après avoir traversé la gaine carpienne, accompagne l'artère palmaire métacarpienne jusqu'au tiers proximal du canon où il se bifurque pour donner les nerfs palmaires. Ces nerfs accompagnent les artères de même nom et se terminent en formant les nerfs collatéraux du doigt correspondant. A propos du nerf cubital, Lesbre (1903) signale qu'il se termine en deux branches au-dessus de l'os pisiforme : une de ces branches innerve les faces externe et palmaire du carpe et l'autre traverse la partie externe de la gaine carpienne. Dans la région métacarpienne, le nerf foumit une branche au ligament suspenseur du boulet, puis se termine audessus de ce ligament par un certain nombre de branches qui accompagnent les artères interosseuses palmaires métacarpiennes. Cauvet (1925) a décrit le plexus brachial et le plexus Iombo-sacré; selon lui, ils sont énormes et le premier d'entre eux est situé à la partie interne de l'épaule. Droandi (1936) se réfère seulement, à ce sujet, à la description de Lesbre (1903).

Les résultats que nous avons obtenus dans nos recherches sont conformes à ceux que donne Lesbre, excepté en ce qui concerne la distribution du nerf cubital et du nerf médian dans la région de la main. De ce fait, notre intention est seulement de signaler ici ces différences.
Nous avons constate que le nerf cubital se termine en une branche superficielle et une branche profonde dans le tiers distal de l'avant-bras. La branche superficieile emerge de l'espace situé sous le tendon du muscle grand palmaire et se termine dans la région du carpe de la façon décrite par Lesbre (1903). La deuxième branche traverse la gaine carpienne en compagnie de l'artère cubitale: Dans le tiers proximal du métacarpe, le nerf se trouve en compagnie de l'artère palmaire métacarpienne moyenne et descend le long du bord latéral des tendons fléchisseurs. Dans la partie distale du métacarpe, le nerf se termine en s'unissant à la branche palmaire externe du nerf médian.

On a pu suivre le trajet du nerf médian à travers la gaine carpienne en compagnie de l'artère palmaire métacarpienne (artère métacarpienne superficielle) sous l'insertion du fléchisseur superficiel des doigts (perforé) à la partie médiane du carpe. Un peu au-dessous du milieu du métacarpe, le nerf médian qui est situé, comme l'artère palmaire métacarpienne superficielle, à la surface palmaire des tendons fléchisseurs, se termine en deux branches palmaires. Une de ces branches, la palmaire externe, est rejointe tout près de son origine par la branche profonde du nerf cubital, comme nous l'avons déjà dit. Chacune de ces branches palmaires du nerf médian se divise, après un très court trajet, en une branche centrale et une branche excentrique. Les branches centrales des deux nerfs s'unissent pour former un tronc nerveux commun comparable au nerf digital commun des bovins. Ce tronc nerveux accompagne l'artère palmaire métacarpienne superficielle (digitale commune), en avant, jusqu'à l'extrémité distale de l'os métacarpien principal. Il se divise bientôt pour former les nerfs palmaires digitaux., interne et externe, des doigts III et IV. Chacun des nerfs palmaires digitaux internes suit le bord frontal de lartère digitale palmaire correspondante. Vers le milieu du bord interne de la seconde phalange, chacun de ces nerfs se termine en deux branches : l'une en avant et l'autre en arrière de l'artère qui 
les accompagne el avec laquelle elles se dirigent jusqu'à la troisieme phalange. Par ailleurs, les branches excentriques descendent obliquement en

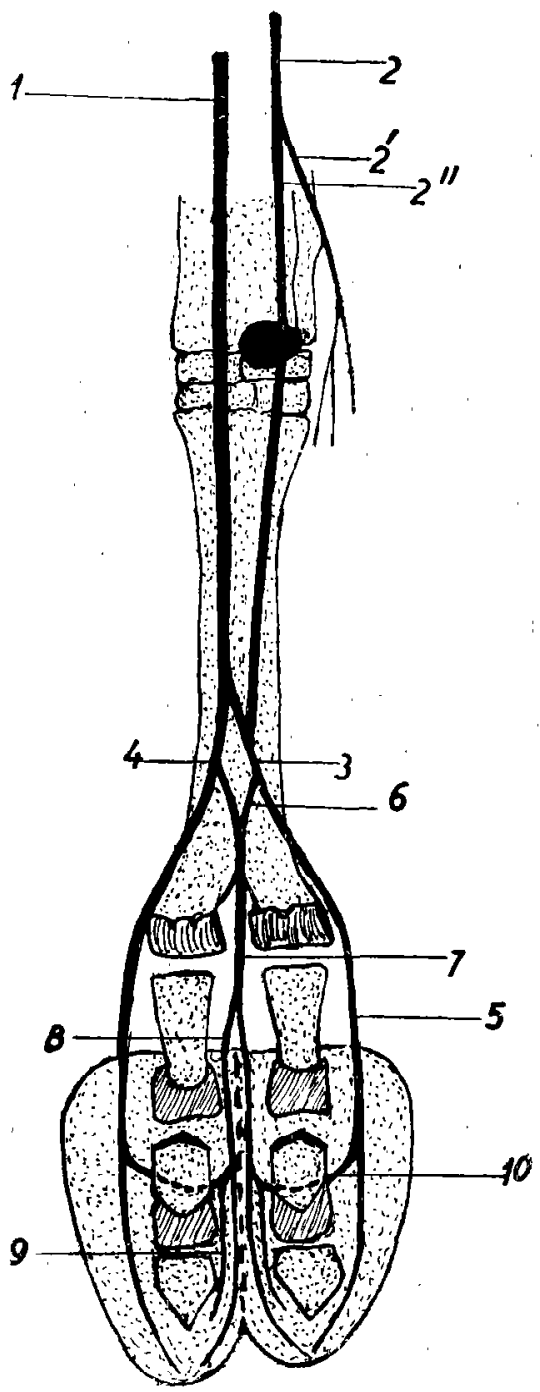

Face palmaire de la région de la main du chameau.

Schema montrant la distribution des nerfs.

1. - Médian;

2. - Cubital;

$2^{\prime}$ - Rameau superficiel du Cubital;

$2^{\prime \prime}$ - Rameau profond du Cubital;

3. - Ramcau palmairc cxterne du Médian;

4. - Rameau palmaire interne du Médian;

5. - Nerf digital palmaire externe;

6. - Rameau central;

7. - Tronc nerveux digital commun;

8. - Nerf digital palmaire interne;

9. - Rameau antérieur du précédent;

10. - Rameau d'anastomose du rameau précédent. se dirigeant légèrement vers l'avant sur la face externe du boulet comme les nerfs digitaux palmaires externes des doigts III et IV.

En partie distale, chacun de ces derniers nerfs accompagne l'artère correspondante jusqu'à la région de la troisième phalange. Finalement, chacun des nerfs digitaux palmaires externes reçoit une anastomose venant du rameau antérieur du nerf palmaire digital interne du même doigt, qui croise la face dorsale de la seconde phalange.

\section{DISCUSSION}

Il est évident, d'après les constatations précédentes, que la partie inférieure du membre antérieur du chameau, jusqu'au carpe, est innervée, comme chez les équins, par le médian et le cubital. Cependant, chez le cheval, l'union entre la branche profonde du cubital et la branche palmaire externe du médian se fait au-dessus du carpe tandis que, chez le chameau, cette union se produit dans le tiers distal du métacarpe. En outre, la disposition de ces nerfs dans la région des doigts chez le chameau ressemble davantage à celle qui existe chez le bœuf qu'à celle qu'on observe chez le cheval. Il faut noter toutefois que les doigts III et IV du chameau reçoivent toute leur innervation du médian et du cubital, alors que les doigts correspondants du boeuf sont en outre innervés par le rameau superficiel du cubital et par les branches cutanées dorsales du nerf radial. Ces nerfis ne descendent pas au-dessous du carpe chez le chameau et leur absence est probablement compensée, dans la région digitale, par les rámeaux antérieurs des nerfs palmaires digitaux internes et leur anastomose avec les nerfs correspondants palmaires digitaux externes, comme on l'a dejà décrit. On peut aussi ajouter que, en raison de l'union du rameau profond du cubital, avec la branche palmaire externe du médian, au lieu de sa branche digitale palmaire externe comme c'est le cas chez le boeuf, il existe évidemment unc dissernblance entre les régions des doigts qui sont innervées par ces nerfs chez ces deux animaux.

\section{B . - LE SYSTÈME MUSCULAIRE}

Lesbre (1903): a établi que le muscle accessoire du grand dorsal ou long extenseur de' l'avantbras (Tonsor fascia antibrachii) est absent chez le chameau. Lombardini [cité par Cauvet '(1925)] a signalé la présence de ce muscle, bien développé, joint à la partie, allongée du muscle triceps du bras. Lesbre (1903) et Cauvet (1925) ont mentionnél'absence de l'anconé et décrit un quadriceps à la place du triceps brachial, chez le chameau. Ils ajoutent que la partie intermédiaire de ce muscle est prise pour 
l'anconé. Ces deux auteurs ont aussi établi que le muscle capsulaire de l'épaule est beaucoup plus développé chez le chameau que chez les solipèdes mais ils ne donnent aucune indication complementaire.

Lesbre (1903) nie que le cubital interne soit absent chez le chameau. Il ajoute que ce muscle a échappé à l'attention des autres observateurs parce qu'il est réduit à un mince tendon, à petite insertion, qui prend naissance sur l'épitrochlée de l'humérus et le cubitus. Il est entouré par les muscles cubital externe et perforé et son tendon est confondu avec celui du cubital externe un peu au-dessus du pisiforme. Cet auteur en conclut que les autres observateurs ont confondu le cubital interne et le perforé.

Cauvet (1925) a signalé l'union du perforé et de la branche radiale du perforant par une bandelette de renforcement et tandis que Lesbre (1903) confirme l'existence de cette union entre les muscles précédents, il ne dit pas de quelie façon elle s'opère.

Lesbre (1903) considère que l'insertion du cubital externe se fait en partie sur le pisiforme et en partie sur le bord externe du carpe. Il ajoute aussi que l'insertion $d u$ grand palmaire se fait sur le bord interne de la partie supérieure du métacarpe et qu'il n'existe pas de rond pronateur chez le chameau. Chez les ruminants ce dernier muscle est représenté par quelques fibres musculaires qui sont enchâssées dans le ligament latéral interne de l'articulation du coude.

Tous les auteurs précédents donnent comme origine du ligament suspenseur du boulet le radius, le pisiforme, la partie distale du carpe et l'extrémité proximale du métacarpe.

En examinant le système musculaire du membre antérieur du chameau à la lumière des descriptions données par les auteurs précédents, parmi lesquels seul Lesbre (1903) a étudié complètement le système musculaire. nous avons pu faire quelques remarques.

De même que pour le système nerveux du membre. précédemment étudié, nous avons l'intention de ne signaler ici que les différences observées en y ajoutant les observations qui pourraient servir à éclairer d'autres points.

Les résultats obtenus ont confirmé l'absence du muscle accessoire du grand dorsal, comme l'avait signalé Lesure (1903), el contredisent l'affirmation. de Lombardini [cité par Cauvet (1925)] à ce sujet. Cependant, il faut ajouter que le tendon commun du grand rond et du grand dorsal, inséré sur la ligne interne de l'humérus, et qui chez d'autres animaux se confond avec le tendon aponévrotique du muscle accessoire du grand dorsal, s'insère chez le chameau sur l'aponévrose antibrachiale et la pointe du cubitus. Ce faisant, cette insertion aponévrotique sur les os de l'avant-bras couvre la surface interne de la branche principale du triceps brachial, comme chez le cheval. Nous avons constaté que le muscle capsulaire de l'épaule est de façon remarquable, plus développé que le muscle correspondant du cheval. Chez le chameau, cependant, ce muscle repose sur la partie postéro-interne de la capsule articulaire de l'épaule, parallèlement à la limite antérieure du muscle grand rond, et s'attache à proximité de l'insertion de ce dernier muscle sur la partie interne de l'humérus. Le muscle capsulaire, chez le cheval, est situé sur la surface de flexion de l'articulation de l'épaule et s'insère sur la face postérieure de l'humérus, un peu au-dessous de sa tête.

Nous avons constaté qu'il existe chez le chameau un muscle semblable à l'anconé des autres animaux par sa position et ses relations avec le diverticulum de la capsule articulaire du coude. Ce muscle est plus développé et se sépare plus aisément de la branche externe du triceps brachial que le muscle homologue du cheval.

La dissection a aussi révélé la présence du muscle cubital interne lel quu'il esl décril par. Lesłore (1903) mais avec un tendon qui se fusionne, sur toute sa longueur, avec le bord postérieur du fléchisseur superficiel des doigts (perforé). C'est, seulement un peu au-dessus du carpe que le tendon du cubital interne se sépare de celui du perforé et va s'insérer sur le pisiforme en même temps que le cubital externe.

Contrairement à ce que dıl Cauvel (1925) sur l'union qui existerait entre le fléchisseur superficiel des doigts et la branche radiale du fléchisseur profond des doigts (perforant) au moyen d'une bandelette de renforcement, nous avons constaté que cette union se fait au moyen d'une courte bandelette musculaire fusiforme, portion du perforé, qui va à la branche humérale et non à la branche radiale du perforant, à proximité de l'union des trois branches de ce muscle en un tendon commun au-dessus du carpe.

En ce qui concerne les insertions du cubita] externe et du cubital interne, on peut ajouter que le premier de ces muscles s'insère sur le pisiforme, l'os carpien pyramidal et le ligament latéral du carpe tandis que le deuxieme a un tendon pourvu d'une synoviale qui traverse la gaine carpienne en partie interne, d'une autre façon que chez le cheval, pour atteindre son insertion comme le mentionne Lesbre. Chez le chameau nous avons constaté que, en pius d'une branche radiale prenant son origine sur toute la face palmaire du radius et du cubitus, le fléchisseur profond des doigts - qui est remarquablement développé chez cet animal - possède aussi une branche musculaire mince qui part du condyle de l'humérus, s'unit en partie avec le, bord antérieur 
du ligament latéral interne et va fusionner en partie distale avec le muscle principal.

Finalement, une dissection soigneuse a révélé que le ligament suspenseur du boulet, comme chez les solipèdes et les bovins, n'a pas d'attache directe sur le radius comme l'ont prétendu d'autres auteurs.

\section{DrsCUSSION}

Selon Sisson (1948), le muscle accessoire du grand dorsal du boeuf est un muscle allongé qui s'étend le long du bord postérieur du triceps brachial. Chez le cheval, la portion musculaire de ce muscle est mince et s'étend surtout sur la partie interne du triceps brachial. Son origine supérieure se confond avec l'insertion tendineuse du grand dorsal et son insertion inférieure aponévrotique aver l'aponévrose antibrachiale et l'olécrane.

De ce fait, on peut conclure que, chez le chameau, la portion chamue du muscle a disparu el que le muscle est représenté seulement par l'attache aponévrotique du grand dorsal sur l'aponévrose antibrachiale et la partie olécranienne du cubitus, attache qui a une position semblable sur la partie interne de la branche principale du triceps brachial, et par des attaches sur l'insertion aponévrotique de l'accessoire du grand dorsal semblables aussi à celles qui existent chez les autres animaux.

Bien que le muscle capsulaire de l'épaule existe chez le cheval et le chameau, il varie en taille et en position chez ces deux animaux. Selon Sisson (1948), ce muscle n'existe pas chez le bœuf.

On a déjà dit que Lesbre a décrit chez le chameau un quadriceps à la place du triceps brachial. Il affirme l'absence du petit anconé et ajoute qu'on a pris la partie intermédiaire du quadriceps pour celui-ci. Il nous semble injustifiable d'accepter les vues de Lesbre à ce sujet car ce qu'il a décrit comme partie intermédiaire du quadriceps ressemble, en fait, au petit anconé des autres animaux.

En ce qui concerne le cubital interne il n'y a rien à ajouter si ce n'est pour confirmer sa présence chez le chameau sous la forme qu'on a décrite précédemment.

On a déjà parlé de l'union entre le perforé et le perforant. Une telle union existe aussi chez le bouf bien qu'elle ne se fasse pas exactement de la même façon. Puisque chez le chameau et chez le boeuf il n'existe pas de brides d'arrêt inférieures pour les tendons des fléchisseurs profonds des doigts, il semble que ce dispositif d'union assume, chez ces deux animaux, une fonction en quelque sorte semblable à celle de ces brides chez le cheval.

Dans l'exposé de Lesbre il est dit que le rond pronateur n'existe pas chez le chameau. Il est question aussi de l'origine humérale, peu marquée du reste, du chef radial du fléchisseur profond des doigts. D'après la précédente description de l'origine musculaire humérale peu sensible de la branche radiale du fléchisseur des doigts, il y a quelques preuves permettant d'avancer que cette origine musculaire humérale est un vestige du rond pronateur chez le chameau. Il est aussi permis d'attribuer le caractère vestigial de ce muscle à la fusion précoce et complète des os de l'avant-bras en position de pronation permanente.

En conclusion, contrairement aux affirmations de précédents auteurs, nos constatations nous permettent de contester que le suspenseur du boulet ait son origine sur le radius. En réclinant vers le haut le ligament pahmaire carpien à partir de son attache distale sur le métacarpe, on a mis à découvert les origines du ligament suspenseur du boulet à partir du pisiforme, de la rangée distale du carpe et de l'extrémité proximale du métacarpe seulement. C'est a cause de la fusion ou de l'intrication du ligament suspenseur avec le ligament palmaire carpien, en arrière de l'extrémité supérieure du métacarpe, que le ligament suspenseur paraît à première vue avoir une attache directe sur le radius. Donc le ligament suspenseur du boulet a, chez le chameau, les mêmes origines que chez les équins et bovins et, en outre, il part aussi du pisiforme.

\section{RÉSUMÉ}

Nous donnons une description détaillée du trajet et de la distribution du nerf médian et du nerf cubital dans la région de la main.

Nous avons constaté que la façon dont ces nerfs se terminent est différente de celle qu'ont décrite d'aulres auteurs.

Nous avons tenté de comparer l'innervation de la région du pied chez le cheval, le boeuf et le chameau.

La signification de l'union qui existe entre le nerf médian et le nerf cubital, à différents niveaux, est envisagée dans le cas du bœuf et dans celui du chameau.

Nous confimons l'absence du muscle accessoire du grand dorsal et la présence du cubital interne. Une suggestion est faite, relative à l'élément qui représente le premier de ces muscles.

Les relations du muscle capsulaire avec I'articulation scapulo-humérale du chameau sont décrites et comparées à celles qui existent chez le cheval. On a constaté que, chez le chameau, le muscle capsulaire de l'épaule est bien plus dévelopipé que chez le cheval.

La signification de l'union entre les fléchisseurs superficiel et profond des doigts, chez le boeuf et chez le chameau, est envisagée à la lumière d'une 
comparaison avec un dispositif different existant chez le cheval.

La preuve de la présence chez le chameau d'un rond pronateur vestigial a été trouvée dans l'origine humérale du chef radial du fléchisseur profond des doigts.

L'origine du ligament suspenseur du boulet sur le radius, affirmée par d'autres chercheurs, n'a pu etre confirmée et nous en donnons les raisons.

\section{BIBLIOGRAPHIE}

CAUVET (1925). - Le chameau. DROANDI (I.) (1936). - Il Camello.

LESBRE (M. F. X.) (1903). - Recherches anatomiques sux les camélidés.

LOMBARDINI, cité par CAUVET (1925). - Le chameau .

SISSON (S.) et GROSSMAN (J. D.) (1948). - The anatomy of domestic animals: 